\title{
PROBABILISTIC CONVERGENCE SPACES
}

\author{
G. D. RICHARDSON and D. C. KENT
}

(Received 9 November 1994; revised 16 August 1995)

Communicated by J. A. Hillman

\begin{abstract}
A basic theory for probabilistic convergence spaces based on filter convergence is introduced. As in Florescu's previous theory of probabilistic convergence structures based on nets, one is able to assign a probability that a given filter converges to a given point. Various concepts and theorems pertaining to convergence spaces are extended to the realm of probabilistic convergence spaces, and illustrated by means of examples based on convergence in probability and convergence almost everywhere. Diagonal axioms due to Kowalsky and Fischer are also studied, first for convergence spaces and then in the setting of probabilistic convergence spaces.
\end{abstract}

1991 Mathematics subject classification (Amer. Math. Soc.): 54A20, 54E70, 60A05.

Keywords and phrases: Probabilistic convergence space, initial probabilistic convergence structure, probabilistic closure operator, Kowalsky diagonal axiom, Fischer diagonal axiom.

\section{Introduction}

Probabilistic metric and topological spaces have been studied extensively during the past thirty years; background information can be found in [4, 8 and 9]. However, it is well known that many types of convergence which arise naturally in probability, statistics, and analysis are non-topological. In 1989, Florescu [3] initiated a study of 'probabilistic convergence structures' based on net convergence. We believe that a more satisfactory theory can be developed in terms of filter convergence, and this paper gives an introduction to such a theory.

We define a probabilistic convergence structure on a set $X$ to be a function $\mathbf{q}$ mapping $\mathbf{F}(X) \times I$ into $2^{X}$, where $\mathrm{F}(X)$ is the set of all filters on $X, I$ is the closed interval $[0,1]$ in $R$, and $2^{X}$ is the power set of $X$; certain additional conditions are also imposed. Essentially, it is convenient to think of $q$ as a family of convergence structures $\left\{q_{\lambda}: \lambda \in I\right\}$; if a filter $\mathscr{F} q_{\lambda}$-converges to $x$, we say that 'the probability

(C) 1996 Australian Mathematical Society 0263-6115/96 \$A2.00+0.00 
that $\mathscr{F} \mathbf{q}$-converges to $x$ is at least $\lambda$ '. Thus $\mathbf{q}$ gives us a rule for determining the probability that any given filter on $X$ converges to any given point in $X$. These notions are made more precise in Section 2.

Section 1 begins with a review of some basic definitions, notations, and terminology pertaining to convergence spaces. We then investigate conditions $K$ and $F$ in the setting of convergence spaces. The former was introduced by Kowalsky in [7], the latter by Fischer in some unpublished notes written around 1974. We answer a question raised by Fischer concerning the possible equivalence of these conditions by showing that a convergence space satisfying $F$ is necessarily topological, whereas one satisfying $K$ is generally not. We also show that $K$, unlike $F$, is not preserved under formation of initial structures (except in the special case where the generating functions are all injective).

In Section 2 we define probabilistic convergence spaces and extend such familiar convergence properties as regularity, first countability, and local compactnesss to such spaces. We also give three examples, two of which arise naturally from real analysis, which illustrate these properties. By considering 'constant' probabilistic convergence spaces, we observe that all of convergence space theory is included within the realm of probabilistic convergence space theory.

In Section 3, we extend the diagonal conditions $K$ and $F$ to a probabilistic convergence space equipped with a ' $t$-norm' $T$ (see [8]). If $(X, \mathbf{q}, T)$ satisfies the Kowalsky (respectively, Fischer) axiom, it is called a Kowalsky (respectively, Fischer) probabilistic convergence space. We show that every Fischer probabilistic convergence space is pretopological, and indeed for pretopological probabilistic convergence spaces the two axioms are equivalent (relative to any fixed $t$-norm). We show that Fischer probabilistic convergence spaces are not always topological except in the case when a certain restriction is placed on the $t$-norm. The same three examples studied in Section 2 are used in Section 3 to shed further light on the significance and applicability of the diagonal conditions.

\section{Convergence spaces and diagonal properties}

Let $X$ be a set, $\mathbf{F}(X)$ the set of all (proper) filters on $X$, and $2^{X}$ the set of all subsets of $X$. For $x \in X$, let $\dot{x}$ be the fixed ultrafilter generated by $\{x\}$. For $\mathscr{F}, \mathscr{G} \in \mathbf{F}(X)$, we write $\mathscr{F} \leq \mathscr{G}$ if and only if $\mathscr{F} \subseteq \mathscr{G}$.

DEFINITION 1.1. A convergence structure $q$ on a set $X$ is a function $q: \mathbf{F}(X) \rightarrow 2^{X}$ satisfying:

$\left(C_{1}\right) \quad x \in q(\dot{x})$, for all $x \in X$;

$\left(C_{2}\right) \quad \mathscr{F} \leq \mathscr{G}$ implies $q(\mathscr{F}) \subseteq q(\mathscr{G})$; 
$\left(C_{3}\right) \quad x \in q(\mathscr{F})$ implies $x \in q(\mathscr{F} \cap \dot{x})$.

The statement $x \in q(\mathscr{F})$ means ' $\mathscr{F} q$-converges to $x$ ', which will usually be written ' $\mathscr{F} \stackrel{q}{\longrightarrow} x$ '. If $q$ is a convergence structure on $X$, then $(X, q)$ is a convergence space.

Let $\mathbf{C}(X)$ be the set of all convergence structures on $X$, partially ordered by: $p \leq q$ if and only if $q(\mathscr{F}) \subseteq p(\mathscr{F})$, for all $\mathscr{F} \in \mathbf{F}(X)$. Relative to this order, $\mathbf{C}(X)$ is a complete lattice whose largest member is the discrete topology $\delta$ on $X$ and whose least member is the indiscrete topology $\iota$.

With each convergence space $(X, q)$, there is an associated closure operator $c l_{q}$ and an associated interior operator $I_{q}$; these are defined for each $A \in 2^{X}$ as follows:

$$
\begin{aligned}
c l_{q} A & =\{x \in X: \exists \mathscr{F} \stackrel{q}{\longrightarrow} x \text { such that } A \in \mathscr{F}\}, \\
I_{q} A & =\{x \in A: \mathscr{F} \stackrel{q}{\longrightarrow} x \text { implies } A \in \mathscr{F}\} .
\end{aligned}
$$

If $\mathscr{F}$ is a filter on $X, c l_{q} \mathscr{F}$ denotes the filter generated by $\left\{c l_{q} F: F \in \mathscr{F}\right\}$. At each $x \in X$, let $\mathscr{V}_{q}(x)=\left\{V \subseteq X: x \in I_{q} V\right\} ; \mathscr{V}_{q}(x)$ is called the $q$-neighborhood filter at $x$. It can also be described as the intersection of all filters which $q$-converge to $x$.

We consider three additional convergence axioms:

$\left(C_{4}\right) \quad q(\mathscr{F} \cap \mathscr{G})=q(\mathscr{F}) \cap q(\mathscr{G})$, for all $\mathscr{F}, \mathscr{G} \in \mathbf{F}(X)$;

$\left(C_{5}\right)$ For each $\mathscr{F} \in \mathbf{F}(X), x \in q(\mathscr{F})$ if and only if $x \in q(\mathscr{G})$, for every ultrafilter $\mathscr{G} \geq \mathscr{F}$

(C $\left.C_{6}\right) \quad x \in q\left(\mathscr{V}_{q}(x)\right)$, for all $x \in X$.

A convergence structure which satisfies $\left(C_{4}\right)$ (respectively, $\left.\left(C_{5}\right),\left(C_{6}\right)\right)$ is called a limit structure (respectively, pseudo-topology, pretopology). Note that pretopology implies pseudo-topology implies limit structure implies convergence structure. A pretopology $q$ is a topology if each neighborhood filter $\mathscr{V}_{q}(x)$ has a filter base of sets which are $q$-open in the sense that the set equals its own interior. A topology can also be characterized as a convergence structure satisfying diagonal condition $F$ (see Corollary 1.5).

A closure operator $c l$ on $X$ is a set function $c l: 2^{X} \rightarrow 2^{X}$ satisfying:

(cl) $\quad c l \emptyset=\emptyset$;

(cl) $A \subseteq c l A$, for $A \in 2^{X}$;

$\left(c l_{3}\right) \quad c l(A \cup B)=\operatorname{cl}(A) \cup \operatorname{cl}(B)$, for all $A, B \in 2^{X}$.

It is well known that for any non-empty set $X$, there exists a bijection between the set of all pretopologies on $X$ and the set of all closure operators on $X$, given by $p \leftrightarrow c l_{p}$, where $c l_{p}$ is the closure operator for the pretopology as defined above. In the next section, we shall extend this to a bijection between 'pretopological probabilistic convergence structures' on $X$ and 'probabilistic closure operators' on $X$. 
We next recall the definitions of some familiar convergence properties. A convergence space $(X, q)$ is said to be:

$T_{1} \quad$ if $q(\dot{x})=\{x\}$, for all $x \in X$;

$T_{2}$ (or Hausdorff) if $q$-convergent filters have unique limits;

Regular if $x \in q\left(\operatorname{cl}_{q} \mathscr{F}\right)$ whenever $x \in q(\mathscr{F})$;

$T_{3}$ if $(X, q)$ is both $T_{1}$ and regular;

First countable if, whenever $x \in q(\mathscr{F})$, there is $\mathscr{G} \in \mathbf{F}(X)$ with a countable filter base such that $x \in q(\mathscr{G})$ and $\mathscr{G} \leq \mathscr{F}$;

Compact if every ultrafilter is $q$-convergent;

Locally compact if every convergent filter contains a compact set.

Let $X$ be a set, $\left\{\left(Y_{\alpha}, p_{\alpha}\right): \alpha \in A\right\}$ a family of convergence spaces, and $f_{\alpha}: X \rightarrow Y_{\alpha}$ a function, for all $\alpha \in A$. The initial convergence structure $q^{w}$ on $X$ induced by $\left\{\left(Y_{\alpha}, p_{\alpha}\right): \alpha \in A\right\}$ and $\left\{f_{\alpha}: \alpha \in A\right\}$ is the coarsest such that $f_{\alpha}:\left(X, q^{w}\right) \rightarrow\left(Y_{\alpha}, p_{\alpha}\right)$ is continuous, for all $\alpha \in A$. It is well known that $q^{w}$ can be characterized as follows: $\mathscr{F} \stackrel{q^{w}}{\longrightarrow} x$ in $X$ if and only if $f_{\alpha}(\mathscr{F}) \stackrel{p_{\alpha}}{\longrightarrow} f_{\alpha}(x)$ in $Y_{\alpha}$, for all $\alpha \in A$. It is also well known that the properties topological, pretopological, pseudo-topological, and regular are initial properties (that is, properties closed under formation of initial structures). The convergence structures defined for subspaces, products, and suprema in $\mathbf{C}(X)$ are all special cases of initial structures relative to appropriate families of spaces and functions.

From the preceding discussion of initial structures it follows that for any $q \in$ $\mathrm{C}(X)$, there is a finest pseudo-topology $v q$ (respectively, pretopology $\pi q$, topology $\tau q$, regular convergence structure $\rho q$ ) coarser than $q$, which is called the pseudotopological (respectively, pretopological, topological, regular) modification of $q$. Obviously, $\tau q \leq \pi q \leq v q \leq q$; there is in general no predictable relationship between $\rho q$ and the other three modifications of $q$.

The notion which is dual to initial structure is called 'final structure'. Let $Y$ be a set, $\left\{\left(X_{\alpha}, q_{\alpha}\right): \alpha \in A\right\}$ a set of convergence spaces, and $f_{\alpha}: X_{\alpha} \rightarrow Y$ a function, for all $\alpha \in A$. The final convergence structure $p^{s}$ on $Y$ induced by $\left\{\left(X_{\alpha}, q_{\alpha}\right): \alpha \in A\right\}$ and $\left\{f_{\alpha}: \alpha \in A\right\}$ is the finest convergence structure on $Y$ such that $f_{\alpha}:\left(X_{\alpha}, q_{\alpha}\right) \rightarrow\left(Y, p^{s}\right)$ is continuous, for all $\alpha \in A$. The final convergence structure is characterized as follows: If $y \in \bigcup\left\{f_{\alpha}(X): \alpha \in A\right\}$, then $\mathscr{F} \stackrel{p^{s}}{\longrightarrow} y$ in $Y$ if and only if there exists $\alpha \in A, x \in f_{\alpha}^{-1}(y)$, and $\mathscr{G} \stackrel{q_{\alpha}}{\longrightarrow} x$ such that $f_{\alpha}(\mathscr{G}) \leq \mathscr{F}$; if $y \in Y-\bigcup\left\{f_{\alpha}(X): \alpha \in A\right\}, \mathscr{F} \stackrel{p^{s}}{\longrightarrow} y$ in $Y$ if and only if $\mathscr{F}=\dot{y}$. It is well known that first countability and local compactness are final properties. The convergence structures for quotient maps, disjoint sums, and infima in $\mathbf{C}(X)$ are all special cases of final structures. Thus for any $q \in \mathbf{C}(X)$, there is a coarsest first countable convergence structure $\sigma q$ finer than $q$, called the first countable modification of $q$ and a coarsest locally compact convergence structure $\gamma q$ finer than $q$, called the locally compact 
modification of $q$.

We next define four 'diagonal conditions' for a convergence space $(X, q)$. For convenience, we introduce the notation $\mathrm{U}(X)$ for the set of all ultrafilters on $X$. If $J$ is any set, $\mathscr{F} \in \mathbf{F}(J)$, and $\sigma: J \rightarrow \mathbf{F}(X)$ is any function, let $\kappa \sigma \mathscr{F}$ be the coarsest filter on $X$ containing all filters of the form $\mathscr{H}_{F}=\bigcap\{\sigma(y): y \in F\}$, for all $F \in \mathscr{F}$. In other words, $\kappa \sigma \mathscr{F}=\sup _{\mathbf{F}(X)}\left\{\mathscr{H}_{F}: F \in \mathscr{F}\right\} . \kappa$ is sometimes called the 'compression operator' for $\sigma$.

$K:$ Let $\sigma: X \rightarrow \mathbf{F}(X)$ be any function such that $\sigma(y) \stackrel{q}{\rightarrow} y$, for all $y \in X$. If $\mathscr{F} \stackrel{q}{\longrightarrow} x$, then $\kappa \sigma \mathscr{F} \stackrel{q}{\longrightarrow} x$.

$K^{*}:$ Let $\sigma: X \rightarrow \mathbf{U}(X)$ be any function such that $\sigma(y) \stackrel{q}{\rightarrow} y$, for all $y \in X$. If $\mathscr{F} \stackrel{q}{\longrightarrow} x$, then $\kappa \sigma \mathscr{F} \stackrel{q}{\longrightarrow} x$.

$F$ : Let $J$ be any set, let $\psi: J \rightarrow X$, and let $\sigma: J \rightarrow \mathbf{F}(X)$ have the property that $\sigma(y) \stackrel{q}{\longrightarrow} \psi(y)$, for all $y \in J$. If $\mathscr{F} \in \mathbf{F}(J)$ is such that $\psi(\mathscr{F}) \stackrel{q}{\longrightarrow} x$, then $\kappa \sigma \mathscr{F} \stackrel{q}{\longrightarrow} x$.

$F^{*}$ : Let $J$ be any set, let $\psi: J \rightarrow X$, and let $\sigma: J \rightarrow \mathrm{U}(X)$ have the property that $\sigma(y) \stackrel{q}{\rightarrow} \psi(y)$, for all $y \in J$. If $\mathscr{F} \in \mathbf{F}(J)$ is such that $\psi(\mathscr{F}) \stackrel{q}{\rightarrow} x$, then $\kappa \sigma \mathscr{F} \stackrel{q}{\longrightarrow} x$.

The diagonal conditions $K$ and $F$ were originally defined by Kowalsky [7] and Fischer (unpublished notes dated 1974), respectively. The two conditions $K^{*}$ and $F^{*}$ are special cases of $K$ and $F$, respectively. Note also that $K$ is a special case of $F$, where $J=X$ and $\psi$ is the identity map on $X$; likewise, $K^{*}$ is a special case of $F^{*}$. These observations are summarized in the next proposition.

PROPOSITION 1.2. For any convergence space $(X, q), K$ implies $K^{*}, F$ implies $F^{*}$ implies $K^{*}$, and $F$ implies $K$.

In [7], Kowalsky showed that if a convergence space $(X, q)$ satisfies $K$, then $\pi q$ is a topology. The next proposition slightly improves this result.

PROPOSITION 1.3. If a convergence space $(X, q)$ satisfies $K^{*}$, then $\pi q$ is a topology.

PROOF. It suffices to show that a convergence structure satisfying $K^{*}$ has the property $\mathrm{Cl}_{q}^{2} A \subseteq \mathrm{cl}_{q} A$ for arbitrary $A \in 2^{X}$. Let $\mathscr{F}$ be an ultrafilter on $X$ containing $c l_{q} A$ such that $\mathscr{F} \stackrel{q}{\longrightarrow} x$. For each $y \in c l_{q} A$, choose an ultrafilter $\mathscr{H}_{y} \stackrel{q}{\longrightarrow} y$ such that $A \in \mathscr{H}_{y}$. We define $\sigma: X \rightarrow \mathbf{U}(X)$ as follows:

$$
\sigma(y)= \begin{cases}\dot{y}, & y \notin c l_{q} A \\ \mathscr{H}_{y}, & y \in c l_{q} A .\end{cases}
$$

Then $\kappa \sigma \mathscr{F} \stackrel{q}{\longrightarrow} x$, and since $c l_{q} A \in \mathscr{F}$ and $A \in \mathscr{H}_{y}$ for all $y \in c l_{q} A, A \in \kappa \sigma \mathscr{F}$. Thus $x \in c l_{q} A$. 
Fischer showed (in unpublished notes) that a pseudo-topology satisfying $F$ is a topology. The next proposition extends this result.

PROPOSITION 1.4. If $(X, q)$ is a convergence space satisfying $F^{*}$, then $q$ is a topo$\log y$.

PROOF. Let $x \in X$, and let $\left\{\mathscr{H}_{\alpha}: \alpha \in J\right\}$ be the set of all ultrafilters $q$-converging to $x$. Define $\psi: J \rightarrow X$ by $\psi(\alpha)=x$, for all $\alpha \in J$, and let $\sigma(\alpha)=\mathscr{H}_{\alpha}$, for all $\alpha \in J$. Let $\mathscr{F}$ be the filter $\{J\}$. Since $\psi(\mathscr{F})=\dot{x} \stackrel{q}{\longrightarrow} x, \kappa \sigma \mathscr{F} \stackrel{q}{\rightarrow} x$, by $F^{*}$. However $\bigcap\{\sigma(y): y \in J\}=\bigcap\left\{\mathscr{H}_{\alpha}: \alpha \in J\right\}=\mathscr{V}_{q}(x) \stackrel{q}{\longrightarrow} x$. Thus $q$ is a pretopology. By Propositions 1.2 and $1.3, q$ is also a topology.

It is well known that a topological space satisfies Condition $F$. Thus we have the following corollaries.

COROLLARY 1.5. For a convergence space $(X, q)$, the following are equivalent. (1) $q$ is a topology; (2) $q$ satisfies $F$; (3) $q$ satisfies $F^{*}$.

COROLLARY 1.6. For a pretopological space $(X, q)$, the following are equivalent. (1) $q$ is a topology; (2) $q$ satisfies $K$; (3) $q$ satisfies $K^{*}$; (4) $q$ satisfies $F$; (5) $q$ satisfies $F^{*}$.

PROPOSITION 1.7. Let $(X, q)$ be a convergence space.

(a) If $(X, q)$ satisfies $K$, then $q$ is a limit structure.

(b) If $(X, q)$ satisfies $K^{*}$, then a finite intersection of ultrafilters $q$-converging to $x$ must also $q$-converge to $x$.

PROOF. The proofs of (a) and (b) are essentially the same, so we prove only (a). Let $\mathscr{F}$ and $\mathscr{G} q$-converge to $x$ and assume $K$. Define

$$
\sigma(y)= \begin{cases}\dot{y}, & y \neq x \\ \mathscr{G} \cap \dot{x}, & y=x .\end{cases}
$$

For $F \in \mathscr{F}, \bigcap\{\sigma(y): y \in F \cup\{x\}\}=\dot{F} \cap \mathscr{G} \cap \dot{x}$, where $\dot{F}$ denotes the filter of oversets of $F$. Thus $\kappa \sigma(\mathscr{F} \cap \dot{x})=\mathscr{F} \cap \mathscr{G} \cap \dot{x}$, which $q$-converges to $x$ by $K$.

The diagonal property $F$ is obviously an initial property, since it is equivalent to the property of being topological. The next proposition gives a partial result in this direction for the properties $K$ and $K^{*}$. 
PROPOSITION 1.8. Let $(X, q)$ be a convergence space, equipped with the initial convergence structure induced by a family $\left\{\left(Y_{\alpha}, p_{\alpha}\right): \alpha \in A\right\}$ of spaces and $\left\{f_{\alpha}: \alpha \in\right.$ A\}, where each $f_{\alpha}: X \rightarrow Y_{\alpha}$ is injective. Then if each $\left(Y_{\alpha}, p_{\alpha}\right)$ satisfies condition $K$ $\left(\right.$ or $\left.K^{*}\right)$, the same is true of $(X, q)$.

PROOF. We prove the result only for $K$; the proof for $K^{*}$ is essentially the same. Let $\mathscr{F} \stackrel{q}{\longrightarrow} x$ and let $\sigma: X \rightarrow \mathbf{F}(X)$ be any function such that $\sigma(y) \stackrel{q}{\longrightarrow} y$, for all $y \in X$. We must verify that $\kappa \sigma \mathscr{F} \stackrel{q}{\longrightarrow} x$. Let $\alpha \in A$ be fixed, and let $\sigma_{\alpha}: Y_{\alpha} \rightarrow \mathbf{F}\left(Y_{\alpha}\right)$ be defined as follows: $\sigma_{\alpha}(y)=\dot{y}$ if $y \in Y_{\alpha}-f_{\alpha}(X), \sigma_{\alpha}(y)=f_{\alpha}\left(\sigma\left(f_{\alpha}^{-1}(y)\right)\right)$ if $y \in f_{\alpha}(X)$. One easily verifies that $f_{\alpha}(\kappa \sigma \mathscr{F}) \geq \kappa \sigma_{\alpha} f_{\alpha}(\mathscr{F})$. The latter filter $p_{\alpha^{-}}$ converges to $f_{\alpha}(x)$ by Condition $K$, and consequently $f_{\alpha}(\kappa \sigma \mathscr{F}) \stackrel{p_{\alpha}}{\longrightarrow} f_{\alpha}(x)$. This holds for all $\alpha \in A$, and so $\kappa \sigma \mathscr{F} \stackrel{q}{\longrightarrow} x$.

We conclude this section with two examples. The first is a limit space which satisfies $K$ but fails to be pretopological, showing that $K$ does not imply $F$. Furthermore, we define a set $X$ and a surjective function $f: X \rightarrow Y$ such that there is no coarsest convergence structure $q$ on $X$ satisfying $K$ such that $f:(X, q) \rightarrow(Y, p)$ is continuous. This shows that the assumption of Proposition 1.8 that the $f_{\alpha}$ 's be injective cannot be dismissed. In other words, unlike $F, K$ is not an initial property.

EXAMPLE 1.9. Let $Y$ be an infinite set, and choose $a \in Y$. Let $\left\{\mathscr{F}_{n}: n \in N\right\}$ be a set of distinct, free ultrafilters on $Y$, and let $\mathscr{G}_{n}=\mathscr{F}_{n} \cap \dot{a}$, for all $n \in N$. We define $p$ to be the finest limit structure on $Y$ such that each $\mathscr{G}_{n} p$-converges to $a$; thus $p$ is not pretopological since $\mathscr{G}=\bigcap\left\{\mathscr{G}_{n}: n \in N\right\}$ does not $p$-converge.

To check that $(Y, p)$ satisfies $K$, assume $\sigma: Y \rightarrow \mathbf{F}(Y)$ is such that $\sigma(y) \stackrel{p}{\longrightarrow} y$, for all $y \in Y$, and let $\mathscr{H} \stackrel{p}{\longrightarrow} x$. If $x \neq a$, then $\mathscr{H}=\dot{x}$ and $\kappa \sigma \mathscr{H}=\sigma(x)=\dot{x}$. If $x=a$, then $\mathscr{H} \geq \bigcap\left\{\mathscr{G}_{n_{i}}: i=1, \ldots, k\right\}$, and one easily checks that $\kappa \sigma \mathscr{H}=\mathscr{H} \cap \sigma(a)$, which $p$-converges to $a$. However, since $p$ is not pretopological, it follows by Proposition 1.4 that $(Y, p)$ does not satisfy $F$.

Next, let $X_{n}=Y \times\{n\}$, and let $X=\bigcup\left\{X_{n}: n \in N\right\}$. Let $f: X \rightarrow Y$ be defined by $f(y, n)=y$, for all $(y, n) \in X$. Let $x_{n}=(a, n)$, for all $n \in N$, and let $q_{n}$ be the finest limit structure on $X$ such that $f^{-1}\left(\mathscr{G}_{k}\right) \stackrel{q_{n}}{\longrightarrow} x_{n}$, for all $k \in N$. The argument of the preceding paragraph shows that $q_{n}$ satisfies $K$, for all $n \in N$. Also note that $f:\left(X, q_{n}\right) \rightarrow(Y, p)$ is continuous, for all $n \in N$.

Finally, suppose there is a coarsest convergence structure $q$ on $X$ satisfying $K$ such that $f:(X, q) \rightarrow(Y, p)$ is continuous. Obviously, $q \leq q_{n}$ for all $n$, and so $f^{-1}\left(\mathscr{G}_{n}\right) \stackrel{q}{\longrightarrow} x_{n}$, for all $n \in N$. To see that $q$ does not satisfy $K$, define $\sigma: X \rightarrow \mathbf{F}(X)$ as follows:

$$
\sigma(z)= \begin{cases}f^{-1}\left(\mathscr{G}_{n}\right), & \text { if } z=x_{n} \text { for some } n \in N \\ \dot{z}, & \text { otherwise. }\end{cases}
$$


Let $\mathscr{F}=f^{-1}\left(\mathscr{G}_{1}\right)$; then $\mathscr{F} \stackrel{q_{1}}{\longrightarrow} x_{1}$. Let $F=f^{-1}(G) \in \mathscr{F}$, where $G \in \mathscr{G}_{1}$. Since $x_{n} \in f^{-1}(G)$, for all $n \in N, \mathscr{K}=\bigcap\left\{f^{-1}\left(\mathscr{G}_{n}\right): n \in N\right\} \geq \bigcap\{\sigma(z): z \in F\}$, and therefore $\mathscr{K} \geq \kappa \sigma \mathscr{F}$. If $\kappa \sigma \mathscr{F} \stackrel{q}{\longrightarrow} x_{1}$, then $f(\mathscr{K})=\mathscr{G} \stackrel{p}{\longrightarrow} a$, a contradiction. Thus $\kappa \sigma \mathscr{F}$ fails to $q$-converge to $x$, and therefore $q$ does not satisfy $K$.

The second example describes a convergence space which satisfies $K^{*}$ but not $K$, showing that (unlike $F$ and $F^{*}$ ) the axioms $K$ and $K^{*}$ are distinct.

EXAMPLE 1.10. Let $X$ be any infinite set, and let $\mathscr{F}$ and $\mathscr{G}$ be two distinct, free filters on $X$ such that neither is a finite intersection of free ultrafilters. Fix $x_{o} \in X$, and define $q$ to be the finest convergence structure on $X$ such that:

$\mathscr{H} \stackrel{q}{\longrightarrow} x_{o}$ if and only if either there is a finite set of free ultrafilters $\mathscr{G}_{1}, \ldots, \mathscr{G}_{n}$, all finer than $\mathscr{G}$, such that $\mathscr{H} \geq \mathscr{F} \cap \mathscr{G}_{1} \cap \ldots \cap \mathscr{G}_{n} \cap \dot{x}_{o}$, or else there is a finite set of free ultrafilters $\mathscr{F}_{1}, \ldots, \mathscr{F}_{k}$, all finer than $\mathscr{F}$, such that $\mathscr{H} \geq \mathscr{G} \cap \mathscr{F}_{1} \cap \ldots \cap \mathscr{F}_{k} \cap \dot{x}_{o}$.

Note that if $\sigma: X \rightarrow \mathbf{U}(X)$ is such that $\sigma(x) \stackrel{q}{\rightarrow} x$ for all $x$, then $\kappa \sigma\left(\mathscr{F} \cap \dot{x_{o}} \cap\right.$ $\left.\mathscr{G}_{1} \cap \cdots \cap \mathscr{G}_{n}\right) \geq \mathscr{F} \cap \dot{x}_{0} \cap \mathscr{G}_{1} \cap \ldots \cap \mathscr{G}_{n} \cap \mathscr{K}$, where $\mathscr{K}$ is some free ultrafilter finer than $\mathscr{G}$; a similar observation applies to $\kappa \sigma\left(\mathscr{G} \cap \dot{x}_{o} \cap \mathscr{F}_{1} \cap \cdots \cap \mathscr{F}_{k}\right)$. Thus $\mathscr{H} \stackrel{q}{\rightarrow} x_{o}$ implies $\kappa \sigma \mathscr{H} \stackrel{q}{\longrightarrow} x_{o}$, and it follows that $(X, q)$ satisfies $K^{*}$. But $(X, q)$ is not a limit space, so $(X, q)$ fails to satisfy $K$, by Proposition 1.7 .

Finally, we remark that none of the diagonal properties are preserved under final structures, since every convergence space is the image of a topological space under a convergence quotient map (see [5]).

\section{Probabilistic convergence spaces}

Let $I$ denote the closed interval $[0,1]$ in $R$. For a non-empty set $X$, recall that $\mathrm{F}(X)$ is the set of all filters on $X, \mathbf{C}(X)$ the set of all convergence structures on $X$, and $2^{X}$ the power set of $X$.

DEFINITION 2.1. A probabilistic convergence structure $\mathbf{q}$ on $X$ is a mapping $\mathbf{q}$ : $\mathbf{F}(X) \times I \rightarrow 2^{X}$ which satisfies:

$\left(P C S_{1}\right)$ For all $\lambda \in I, \mathbf{q}(\mathscr{F}, \lambda)=q_{\lambda}(\mathscr{F})$, where $q_{\lambda} \in \mathbf{C}(X)$.

$\left(P C S_{2}\right)$ If $\lambda=0, q_{0}$ is the indiscrete topology $\iota$ on $X$.

$\left(P C S_{3}\right)$ If $\mu \leq \lambda$ in $I$, then $q_{\mu} \leq q_{\lambda}$ in $\mathbf{C}(X)$.

If $\mathbf{q}$ is a probabilistic convergence structure on $X$, then $(X, \mathbf{q})$ is called a probabilistic convergence space. We shall use the abbreviation 'p.c.s' ambiguously for both 'probabilistic convergence structure' and 'probabilistic convergence space'; it should be clear from the context which meaning is intended. 
For a p.c.s. $\mathbf{q}$ as in Definition 2.1, it will often be convenient to denote the ' $\lambda$-th component structure' $q_{\lambda}$ by $(\mathbf{q})_{\lambda}$. We interpret ' $x \in q_{\lambda}(\mathscr{F})$ ' to mean 'the probability that $\mathscr{F} q$-converges to $x$ is not smaller than $\lambda$ '. The probability that $\mathscr{F} \mathbf{q}$-converges to $x$ is defined to be $\lambda=\sup \left\{\mu \in I: \mathscr{F} \stackrel{q_{\mu}}{\longrightarrow} x\right\}$. The fact that $(\mathbf{q})_{0}$ is indiscrete guarantees that the set $\left\{\mu: \mathscr{F} \stackrel{q_{\mu}}{\longrightarrow} x\right\}$ is non-empty, since this set necessarily contains 0 .

If $p \in \mathbf{C}(X)$ and $\mathbf{q}$ is a p.c.s. on $X$ such that $(\mathbf{q})_{\lambda}=p$, for all $\lambda \in(0,1]$, then we call $\mathbf{q}$ a constant p.c.s. and denote it by $\mathbf{q}_{p}$.

Let $\mathbf{P C}(X)$ be the set of all p.c.s.'s on $X$, partially ordered by $\mathbf{q} \leq \mathbf{r}$ if and only if $(\mathbf{q})_{\lambda} \leq(\mathbf{r})_{\lambda}$, for all $\lambda \in I$. Clearly, the least member of $\mathbf{P C}(X)$ is the indiscrete p.c.s $\mathbf{q}_{t}$ and the greatest member of $\mathbf{P C}(X)$ is the discrete p.c.s. $\mathbf{q}_{\delta}$. Using the obvious one-to-one correspondence $p \leftrightarrow \mathbf{q}_{p}$ to identify convergence structures with their corresponding constant p.c.s.'s, we can regard $\mathbf{C}(X)$ as a subset of $\mathbf{P C}(X)$. Indeed, $\mathbf{P C}(X)$ is a complete lattice and $\mathbf{C}(X)$ is a sub-complete-lattice of $\mathbf{P C}(X)$. Thus 'probabilistic convergence space' is a simple and direct generalization of 'convergence space'.

Let $(X, \mathbf{q})$ and $(Y, \mathbf{p})$ be p.c.s.'s, and $f: X \rightarrow Y$ a function. Then $f:(X, \mathbf{q}) \rightarrow$ $(Y, \mathbf{p})$ is continuous if $f:\left(X,(\mathbf{q})_{\lambda}\right) \rightarrow\left(Y,(\mathbf{p})_{\lambda}\right)$ is continuous for all $\lambda \in I$. A p.c.s. $(X, \mathbf{q})$ is defined to be pseudo-topological (respectively, pretopological, topological, regular, first countable, compact, locally compact, $\mathbf{T}_{1}, \mathbf{T}_{2}$ ) if, for each $\lambda \in(0,1],\left(X,(\mathbf{q})_{\lambda}\right)$ is pseudo-topological (respectively, pretopological, topological, regular, first countable, compact, locally compact, $\left.\mathbf{T}_{1}, \mathbf{T}_{2}\right)$. We also define $(X, \mathbf{q})$ to be left-continuous if, for each $\lambda \in(0,1], q_{\lambda}=\sup _{\mathbf{C}(X)}\left\{q_{\mu}: \mu<\lambda\right\}$. If $p \in \mathbf{C}(X)$, it is clear that the constant p.c.s. $\mathbf{q}_{p}$ is left-continuous, and all the properties pseudotopological, pretopological, etcetera are possesed by $\mathbf{q}_{p}$ in the p.c.s.-sense if and only if they are possesed by $p$ in the convergence structure sense.

If $(X, \mathbf{q})$ is a p.c.s., the pseudo-topological modification $\nu \mathbf{q}$ of $\mathbf{q}$ is defined by $(v \mathbf{q})_{\lambda}=v q_{\lambda}$ for all $\lambda \in I$, where $v q_{\lambda}$ is the finest pseudo-topology in $\mathbf{C}(X)$ coarser than $q_{\lambda}$. Obviously, $v \mathbf{q}$ is the finest pseudo-topological p.c.s. in $\mathbf{P C}(X)$ coarser than q. We can define the pretopological modification $\pi \mathbf{q}$, the topological modification $\tau \mathbf{q}$, and the regular modification $\rho \mathbf{q}$ of $\mathbf{q}$ in the analogous way.

One may also define the first countable modification $\varphi \mathbf{q}$ of $\mathbf{q}$ by taking $(\varphi \mathbf{q})_{\lambda}=\varphi q_{\lambda}$, where $\varphi q_{\lambda}$ is the coarsest first countable member of $\mathbf{C}(X)$ finer than $q_{\lambda}$. The locally compact modification $\gamma \mathbf{q}$ of $\mathbf{q}$ is defined analoguously.

PROPOSITION 2.2. If $(X, \mathbf{q})$ is a left-continuous p.c.s., so are $\nu \mathbf{q}$ and $\varphi \mathbf{q}$.

Proof. Let $\lambda \in I$ be fixed, and assume $q_{\lambda}=\sup _{\mathbf{C}(X)}\left\{q_{\mu}: \mu<\lambda\right\}$. Assume $\mathscr{F} \stackrel{v q_{\mu}}{\longrightarrow} x$, for all $\mu<\lambda$. Then each ultrafilter $\mathscr{G} \geq \mathscr{F} q_{\mu}$-converges to $x$, for all $\mu<\lambda$, and so $\mathscr{G} \stackrel{q_{\lambda}}{\longrightarrow} x$. Consequently, $\mathscr{F} \stackrel{v q_{\lambda}}{\longrightarrow} x$, and $\operatorname{so} v q_{\lambda}=\sup _{\mathbf{C}(X)}\left\{v q_{\mu}: \mu<\right.$ 
$\lambda\}$.

Next, assume $\mathscr{F} \stackrel{\varphi q_{\mu}}{\longrightarrow} x$, for all $\mu<\lambda$. Choose an increasing sequence $\mu_{n}$ in $[0, \lambda)$ converging to $\lambda$. For each $n$, there is a filter $\mathscr{G}_{n}$ with a countable filter base such that $\mathscr{G}_{n} \stackrel{q_{\mu_{n}}}{\longrightarrow} x$ and $\mathscr{G}_{n} \leq \mathscr{F}$. If $\mathscr{G}=\sup _{\mathbf{F}(X)}\left\{\mathscr{G}_{n}: n \in N\right\}$, then $\mathscr{G}$ has a countable filter base, $\mathscr{G} \stackrel{q_{\lambda}}{\longrightarrow} x$ and $\mathscr{G} \leq \mathscr{F}$. Thus $\mathscr{F} \stackrel{\varphi q_{\lambda}}{\longrightarrow} x$.

We next give three examples, two of which arise naturally in real analysis.

EXAMPLE 2.3. Let $(X, r)$ be a convergence space, and for $\lambda \in I$ define:

$$
q_{\lambda}= \begin{cases}\iota, & \lambda=0 \\ r, & 0<\lambda \leq 1 / 2 \\ \delta, & 1 / 2<\lambda \leq 1 .\end{cases}
$$

Let $\mathbf{q}$ be the p.c.s. defined by $(\mathbf{q})_{\lambda}=q_{\lambda}$, for each $\lambda \in I$. We note that $\mathbf{q}$ is left-continuous, and is pseudo-topological (respectively, pretopological, topological, $\mathbf{T}_{1}, \mathbf{T}_{2}$, regular, $\mathbf{T}_{3}$, first countable, locally compact) if and only if the convergence structure $r$ has the corresponding property. Also note that $\mathbf{q}$ is constant if and only if $r=\delta$, in which case it is the discrete p.c.s. If $x \in X, \mathscr{F} \in \mathbf{F}(X)$, and $\mathscr{F} \neq \dot{x}$, then $\mathscr{F}$ q-converges to $x$ with probability 0 if $\mathscr{F} \stackrel{r}{\rightarrow} x$, and with probability $1 / 2$ if $\mathscr{F} \stackrel{r}{\rightarrow} x$.

EXAMPLE 2.4. Let $l$ denote Lebesgue measure on $I$, and let $\tau$ be the usual topology on $R$. Let $X$ be the set of all real-valued, Lebesgue measurable functions on $I$. Given $\lambda \in I$, define $\mathscr{F} \stackrel{q_{\lambda}}{\longrightarrow} f$ if and only if there is a subset $A$ of $I$ such that $l(A) \leq 1-\lambda$ and $\mathscr{F}(\mu) \stackrel{\tau}{\longrightarrow} f(\mu)$, for all $\mu \in I-A$. The p.c.s. $\mathbf{q}$ is defined on $X$ by $(\mathbf{q})_{\lambda}=q_{\lambda}$, for all $\lambda \in I$. Note that $q_{1}$ describes convergence almost everywhere on $I$ with respect to $l$.

One may verify that $\mathbf{q}$ is regular, since for $\mathscr{F}(\mu) \stackrel{\tau}{\longrightarrow} f(\mu)$, we have $\left(c l_{q_{\lambda}} \mathscr{F}\right)(\mu) \geq$ $c l_{\tau}(\mathscr{F}(\mu))$, and the latter filter $\tau$-converges to $f(\mu)$ by regularity of $\tau$. We shall also verify that $\mathbf{q}$ is left-continuous. Assume $\mathscr{F} \stackrel{q_{\mu}}{\longrightarrow} f$, for all $\mu \in[0, \lambda)$, and let $\mu_{n}$ be an increasing sequence in $I$ converging to $\lambda$. Choose $A_{n}$ such that $l\left(A_{n}\right) \leq 1-\mu_{n}$ and $\mathscr{F}(\mu) \rightarrow f(\mu)$, for all $\mu \in I-A_{n}$. Let $A=\cap\left\{A_{n}: n \in N\right\}$. Then $l(A) \leq \varlimsup_{\lim } l\left(A_{n}\right)$ $\leq 1-\lambda$, and $\mathscr{F}(\mu) \stackrel{\tau}{\longrightarrow} f(\mu)$, for all $\mu \in I-A$. Thus $\mathscr{F} \stackrel{q_{\lambda}}{\longrightarrow} f$, and it follows that $\sup \left\{q_{\mu}: \mu<\lambda\right\}=q_{\lambda}$.

It is obvious that $\mathbf{q}$ is not $\mathbf{T}_{1}$. We shall show that $\mathbf{q}$ is neither pretopological nor locally compact. Let $1_{A}$ denote the characteristic function of $A \subseteq I$. Note that $\dot{\mathrm{i}}_{\{\beta\}} \stackrel{q_{1}}{\longrightarrow} 0$ (the zero function) for each $\beta \in I$, but $\bigcap\left\{\dot{1}_{\{\beta\}}: \beta \in I\right\}$ fails to $q_{1^{-}}$ converge. Thus $q_{1}$ is not pretopological. To see that $q_{1}$ is not locally compact, let $f_{i j}=i 1_{(0,1 / j]}$ and let $F_{n}=\left\{f_{i j}: i \geq 1, j \geq n\right\}$. Let $\mathscr{F}$ be the filter on $X$ whose base is $\left\{F_{n}: n \in N\right\}$. It is easy to see that $\mathscr{F} \stackrel{q_{1}}{\longrightarrow} 0$. However any free ultrafilter containing 
$\left\{f_{i j}: i \geq 1, j\right.$ fixed $\}$ fails to $q_{1}$-converge. Since each member of $\mathscr{F}$ contains a subset of this form, $q_{1}$ is not locally compact.

EXAMPLE 2.5. Let $X$ be the set described in Example 2.4, and let $Y$ be the set obtained from $X$ by identifying functions which are equal almost everywhere. We define a p.c.s. $\mathbf{p}$ on $Y$, where $p_{\lambda}=(\mathbf{p})_{\lambda}$ is defined by setting $p_{0}=\imath$, and, for each $\lambda \in(0,1], \mathscr{F} \stackrel{p_{\lambda}}{\longrightarrow} f$ in $Y$ if and only if, for each $a>0$ and $\epsilon<\lambda$ there exists $F \in \mathscr{F}$ such that, for each $g \in F, l\{\mu \in I:|g(\mu)-f(\mu)|<a\} \geq \epsilon$. For $\lambda=1, p_{1}$ describes convergence in probability, and it is shown in Theorem 4.1.5, [1] that $p_{1}$ is a metrizable topology.

Let $\left(a_{n}\right)$ be a decreasing sequence in the open interval $(0, \lambda)$ converging to 0 , and for $f \in Y$, let $V_{n}(f)=\left\{g \in Y: l\left\{\mu \in I:|g(\mu)-f(\mu)|<a_{n}\right\} \geq \lambda-a_{n}\right\}$. Note that the filter $\mathscr{V}_{p_{\lambda}}(f)$ generated by $\left\{V_{n}(f): n \in N\right\}$ is the $p_{\lambda}$-neighborhood filter at $f$, and this filter obviously has a countable base and $p_{\lambda}$-converges to $f$. Thus $(Y, \mathbf{p})$ is pretopological and first countable. One may also verify that $(Y, \mathbf{p})$ is left-continuous. On the other hand, it is easy to see that $p_{\lambda}$ is not $\mathbf{T}_{1}$ for $\lambda<1$, and it is not difficult to show that $\mathbf{p}$ is neither regular nor locally compact.

Let $X$ be a set, $\left\{\left(Y_{\alpha}, \mathbf{p}^{\alpha}\right): \alpha \in A\right\}$ a set of p.c.s.'s, and $f_{\alpha}: X \rightarrow Y_{\alpha}$ a function, for each $\alpha \in A$. The initial p.c.s. $\mathbf{q}^{w}$ is defined to be the coarsest p.c.s. on $X$ such that $f_{\alpha}:\left(X, \mathbf{q}^{w}\right) \rightarrow\left(Y_{\alpha}, \mathbf{p}^{\alpha}\right)$ is continuous, for all $\alpha \in A$. For each $\alpha \in A$ and $\lambda \in I$, let $\left(\mathbf{p}^{\alpha}\right)_{\lambda}=p_{\lambda}^{\alpha}$. We omit the easy proof of the next proposition.

PROPOSITION 2.6. In the notation of the preceding paragraph, $\left(\mathbf{q}^{w}\right)_{\lambda}=q_{\lambda}^{w}$, where for each $\lambda \in I, q_{\lambda}^{w}$ is the initial convergence structure on $X$ induced by $\left\{f_{\alpha}: \alpha \in A\right\}$, where $f_{\alpha}: X \rightarrow\left(Y_{\alpha}, p_{\lambda}^{\alpha}\right)$, for all $\alpha \in A$. Thus $\mathscr{F} \stackrel{q_{\lambda}^{w}}{\longrightarrow} x$ if and only if $f_{\alpha}(\mathscr{F}) \stackrel{p_{\lambda}^{\alpha}}{\longrightarrow}$ $f_{\alpha}(x)$, for all $\alpha \in A$.

PROPOSITION 2.7. The following p.c.s. properties are initial properties: pseudotopological, pretopological, topological, regular, constant, left-continuous.

PROOF. It was remarked in Section 1 that the first four properties are initial convergence space properties, and the conclusion for p.c.s.'s follows by Proposition 2.6. It is also clear from Proposition 2.6 that the property of being constant is an initial property. Finally, assume the notation of the paragraph preceding Proposition 2.6, and assume $\mathscr{F} \stackrel{q_{\mu}^{w}}{\longrightarrow} x$, for all $\mu<\lambda$. Then $f_{\alpha}(\mathscr{F}) \stackrel{p_{\mu}^{\alpha}}{\longrightarrow} f_{\alpha}(x)$, for all $\mu<\lambda$ and for all $\alpha \in A$. By left-continuity of $\mathbf{p}^{\alpha}, f_{\alpha}(\mathscr{F}) \stackrel{p_{\lambda}^{\alpha}}{\longrightarrow} f_{\alpha}(x)$, for all $\alpha \in A$. Thus $\mathscr{F} \stackrel{q_{i}^{u}}{\longrightarrow} x$, and $q_{\lambda}^{w}=\sup _{\mathrm{C}(X)}\left\{q_{\mu}^{w}: \mu<\lambda\right\}$.

Proposition 2.8. Let $\left(X, \mathbf{q}^{w}\right)$ be the initial p.c.s. induced by $\left\{f_{\alpha}: \alpha \in A\right\}$ and $\left\{\left(Y_{\alpha}, \mathbf{p}^{\alpha}\right): \alpha \in A\right\}$. Given $\mathscr{F} \in \mathbf{F}(X)$ and $x \in X$, let $\lambda$ be the probability that 
$\mathscr{F} \stackrel{\mathbf{q}^{\alpha}}{\longrightarrow} x$. If $\lambda_{\alpha}$ is the probability that $f_{\alpha}(\mathscr{F}) \mathbf{p}^{\alpha}$-converges to $f_{\alpha}(x)$, for all $\alpha \in A$, then $\lambda=\inf \left\{\lambda_{\alpha}: \alpha \in A\right\}$.

PROOF. Let $J=\left\{\mu: \mathscr{F} \stackrel{q_{\mu}^{w}}{\longrightarrow} x\right\}$ and $J_{\alpha}=\left\{\mu: f_{\alpha}(\mathscr{F}) \stackrel{p_{\mu}^{\alpha}}{\longrightarrow} f_{\alpha}(x)\right\}$, for all $\alpha \in A$. Since $J \subseteq J_{\alpha}$ for each $\alpha \in A$ follows by Proposition 2.6, $\lambda=\sup J \leq \lambda_{\alpha}=\sup J_{\alpha}$ holds. If $\eta=\inf \left\{\lambda_{\alpha}: \alpha \in A\right\}>\lambda$, choose $\lambda^{\prime}$ such that $\lambda<\lambda^{\prime}<\eta$. Then $f_{\alpha}(\mathscr{F}) \stackrel{p_{i^{\prime}}^{a}}{\longrightarrow} f_{\alpha}(x)$ holds for all $\alpha \in A$, which implies $\lambda^{\prime} \in J$, a contradiction. Consequently, $\lambda=\inf \left\{\lambda_{\alpha}: \alpha \in A\right\}$.

The most important special cases of initial structures are subspace structures and product structures. If $(Y, \mathbf{p})$ is a p.c.s., $X \subseteq Y$, and $i_{X}: X \rightarrow Y$ is the inclusion map, then the subspace p.c.s. $(X, \mathbf{q})$ is defined by $\mathscr{F} \stackrel{q_{\lambda}}{\longrightarrow} x$ in $X$ if and only if $i_{X}(\mathscr{F}) \stackrel{p_{\lambda}}{\longrightarrow} x$ in $Y$, for all $\lambda \in I$. Thus the probability that $\mathscr{F} \mathbf{q}$-converges to $x$ in $(X, \mathbf{q})$ equals the probability that $i_{X}(\mathscr{F})$ p-converges to $x$ in $(Y, \mathbf{p})$.

For any p.c.s. $(X, \mathbf{q})$, we define a subset $A$ of $X$ to be $q$-closed if $A=c l_{q_{\lambda}} A$, for all $\lambda \in I$.

PROPOSITION 2.9. The following p.c.s. properties are hereditary relative to arbitrary subspaces: $\mathbf{T}_{1}, \mathbf{T}_{2}$, regular, first countable, pseudo-topological, pretopological, topological, left-continuous, and constant. Compactness and local compactness are hereditary relative to $\mathbf{q}$-closed subspaces.

If $(X, \mathbf{q})=\prod\left\{\left(Y_{\alpha}, \mathbf{p}^{\alpha}\right): \alpha \in A\right\}$ is a product p.c.s., then by Proposition $2.8, \mathscr{F}$ $\mathbf{q}$-converges to $x$ with probability $\lambda$ if and only if $\lambda$ is the infimum of the probabilities of the $\mathbf{p}^{\alpha}$-convergence of the projections of $\mathscr{F}$ to the projections of $x$ relative to the various component spaces.

PROPOSITION 2.10. The following p.c.s. properties are preserved under arbitrary products: $\mathbf{T}_{1}, \mathbf{T}_{2}$, regular, compact, pseudo-topological, pretopological, topological, left-continuous, constant. First countability is preserved under countable products, and local compactness under finite products.

We next turn to final probabilistic convergence structures. Let $Y$ be a set, let $\left\{\left(X_{\alpha}, \mathbf{q}^{\alpha}\right): \alpha \in A\right\}$ be a set of p.c.s.'s, and let $f_{\alpha}: X_{\alpha} \rightarrow Y$ be a function, for all $\alpha \in A$. The finest convergence structure $\mathbf{p}^{s}$ on $Y$ such that $f_{\alpha}:\left(X_{\alpha}, \mathbf{q}^{\alpha}\right) \rightarrow\left(Y, \mathbf{p}^{s}\right)$ is continuous for all $\alpha \in A$, is called the final p.c.s. induced by $\left\{f_{\alpha}: \alpha \in A\right\}$ and $\left\{\left(X_{\alpha}, \mathbf{q}^{\alpha}\right): \alpha \in A\right\}$.

PROPOSITION 2.11. In the notation of the preceding paragraph, the final p.c.s. $\mathbf{p}^{s}$ on $Y$ induced by $\left\{f_{\alpha}: \alpha \in A\right\}$ and $\left\{\left(X_{\alpha}, \mathbf{q}^{\alpha}\right): \alpha \in A\right\}$ is characterized as follows: If $y \in \bigcup\left\{f_{\alpha}(X): \alpha \in A\right\}$ and $\lambda>0$, then $\mathscr{F} \stackrel{p_{\lambda}^{s}}{\longrightarrow} y$ if and only if $\exists \alpha \in A$, 
$\exists x \in f_{\alpha}^{-1}(y)$, and $\exists \mathscr{G} \stackrel{q_{\lambda}^{\alpha}}{\longrightarrow} x$ such that $\mathscr{F} \geq f_{\alpha}(\mathscr{G})$; if $y \in Y-\bigcup\left\{f_{\alpha}(X): \alpha \in A\right\}$ and $\lambda>0$, then $\mathscr{F} \stackrel{p_{\lambda}^{s}}{\longrightarrow} y$ if and only if $\mathscr{F}=\dot{y} ; p_{0}^{s}$ is indiscrete.

PROPOSITION 2.12. The following p.c.s. properties are preserved under the formation of p.c.s. final structures: first countable, locally compact, constant.

The most useful final structures are quotient maps and disjoint sums. We shall discuss only the former, although the latter are needed in the proof of Proposition 2.13. A mapping $f:(X, \mathbf{q}) \rightarrow\left(Y, \mathbf{p}^{s}\right)$ is a p.c.s. quotient map if $f$ is onto $Y$ and $\mathscr{F} \stackrel{p_{\lambda}^{s}}{\longrightarrow} y$ in $Y$ if and only if $\exists x \in f^{-1}(y)$ and $\mathscr{G} \stackrel{q_{\lambda}}{\longrightarrow} x$ such that $f(\mathscr{G}) \leq \mathscr{F}$. A p.c.s. is said to be pseudo-metrizable if $(X, \mathbf{q})$ is topological and $q_{\lambda}$ is pseudo-metrizable, for each $\lambda \in I$. The convergence space versions of the two statements of the next proposition are proved in [5 and 6], respectively; these proofs generalize immediately to p.c.s.'s.

PROPOSITION 2.13. (a) Every p.c.s. is the image of a topological p.c.s. under a p.c.s. quotient map.

(b) A p.c.s. is first countable if and only if it is the image of a pseudo-metrizable p.c.s. under a convergence quotient map.

Proposition 2.14. Let $f:(X, \mathbf{q}) \rightarrow(Y, \mathbf{p})$ be a p.c.s. quotient map. Let $\mathscr{F} \in$ $\mathbf{F}(Y)$ and $y \in Y$. If $\mathscr{G} \in \mathbf{F}(X)$ and $x \in X$, let $\lambda_{\mathscr{G}_{, x}}$ be the probability that $\mathscr{G}$ $\mathbf{q}$-converges to $x$. If $\lambda$ denotes the probability that $\mathscr{F} \mathbf{p}$-converges to $y$, then $\lambda=$ $\sup \left\{\lambda_{\mathscr{G}, x}: f(\mathscr{G}) \leq \mathscr{F}\right.$ and $\left.x \in f^{-1}(y)\right\}$.

PROOF. If $f(\mathscr{G}) \leq \mathscr{F}$ and $x \in f^{-1}(y)$, then $\mathscr{G} \stackrel{q_{\mu}}{\longrightarrow} x$, for all $\mu<\lambda_{\mathscr{G}, x}$, which implies $\mathscr{F} \stackrel{p_{\mu}}{\longrightarrow} y$, for all $\mu<\lambda_{G_{,}, x}$. Thus $\lambda_{\mathscr{G}_{, x}} \leq \lambda$. Let $\eta=\sup \left\{\lambda_{\mathscr{G}_{1} x}: f(\mathscr{G}) \leq\right.$ $\left.\mathscr{F}, x \in f^{-1}(y)\right\}$. If $\eta<\lambda$, choose $\lambda^{\prime} \in I$ such that $\eta<\lambda^{\prime}<\lambda$. Then $\mathscr{F} \stackrel{p_{\lambda^{\prime}}}{\longrightarrow} y$, so there exist $x \in f^{-1}(y)$ and $\mathscr{G} \stackrel{q_{\lambda^{\prime}}}{\longrightarrow} x$ such that $f(\mathscr{G}) \leq \mathscr{F}$. This implies $\lambda^{\prime} \leq \lambda_{\mathscr{G}, x}$, contradicting the fact that $\eta<\lambda^{\prime}$.

We conclude this section by giving a characterization of pretopological p.c.s.'s in terms of 'probabilistic closure operators' which extends the familiar relationship between pretopologies and closure operators mentioned in Section 1.

DEFINITION 2.15. A probabilistic closure operator $\mathrm{cl}$ on $X$ is a function $\mathrm{cl}: 2^{X} \times$ $I \rightarrow 2^{X}$ such that :

$\left(p c l_{1}\right)$ For each $\lambda \in I, c l_{\lambda}: 2^{X} \rightarrow 2^{X}$ is a closure operator on $X$, defined by $c l_{\lambda} A=\operatorname{cl}(A, \lambda)$, for all $A \in 2^{X}$.

(pcl $)$ For $\lambda=0, c l_{0} A=X$, for all $A \in 2^{X}-\{\emptyset\}$, and $c l_{0} \emptyset=\emptyset$. 
( $\left.p c l_{3}\right)$ If $\mu \leq \lambda$ in $I$, then $c l_{\lambda} A \subseteq c l_{\mu} A$, for all $A \in 2^{X}$.

A probabilistic closure operator $\mathrm{cl}$ is said to be left-continuous if, for each $\lambda \in(0,1]$ and $A \in 2^{X}, c l_{\lambda} A=\bigcap\left\{c l_{\mu} A: \mu<\lambda\right\}$. We omit the straight-forward proof of the next proposition.

PROPOSITION 2.16. Let $(X, \mathbf{q})$ be a pretopological p.c.s., and define $\mathbf{c l}_{\mathbf{q}}: 2^{X} \times$ $I \rightarrow 2^{X}$ by: $\mathbf{c l}_{\mathbf{q}}(A, \lambda)=\operatorname{cl}_{q_{\lambda}} A$, for each $A \in 2^{X}$ and $\lambda \in I$. Then $\mathbf{c l}_{\mathbf{q}}$ is a probabilistic closure operator which is left-continuous if and only if $(X, q)$ is leftcontinuous. Furthermore, the correspondence $\mathbf{q} \leftrightarrow \mathbf{c l}_{\mathbf{q}}$ is a bijection between the set of all (left-continuous) pretopological p.c.s.'s on X and the set of all (left-continuous) probabilistic closure operators on $X$.

\section{Diagonal probabilistic convergence spaces}

In this section, we consider probabilistic convergence spaces subject to two diagonal conditions derived from the diagonal axioms $K$ and $F$ discussed in Section 1. These diagonal conditions for p.c.s.'s also make use of the notion of 'triangular norm' as defined in [8]. The addition of these diagonal conditions make our filter-based theory conform more closely to the net-based theory of 'probabilistic convergence spaces' of Florescu [3].

DEFINITION 3.1. A triangular norm (or $t$-norm) is a binary operation $T: I^{2} \rightarrow$ $I$ which is associative, commutative, increasing in each variable, and satisfies: $T(a, 1)=a$, for all $a \in I$.

If $T$ and $T^{\prime}$ are $t$-norms, $T \leq T^{\prime}$ means that $T(a, b) \leq T^{\prime}(a, b)$, for all $(a, b) \in I^{2}$. The smallest $t$-norm is $T_{0}$, defined by

$$
T_{0}(a, b)= \begin{cases}a, & \text { if } b=1 \\ b, & \text { if } a=1 \\ 0, & \text { otherwise }\end{cases}
$$

The largest $t$-norm is $T_{1}$, where $T_{1}(a, b)=\min \{a, b\}$, for all $(a, b) \in I^{2}$.

Let $(X, q)$ be a p.c.s. and $T$ a $t$-norm on $X$. Consider the following diagonal conditions on $(X, \mathbf{q})$.

$K_{T}:$ Let $\sigma: X \rightarrow \mathbf{F}(X)$ be any function such that $\sigma(y) \stackrel{q_{\mu}}{\longrightarrow} y$, for all $y \in X$, where $\mu \in I$. If $\lambda \in I$ and $\mathscr{F} \stackrel{q_{\lambda}}{\longrightarrow} x$, then $\kappa \sigma \mathscr{F} \stackrel{q_{T(\lambda, \mu)}}{\longrightarrow} x$.

$F_{T}$ : Let $J$ be any non-empty set, let $\psi: J \rightarrow X$, and let $\sigma: J \rightarrow \mathbf{F}(X)$ be such that $\sigma(y) \stackrel{q_{\mu}}{\longrightarrow} \psi(y)$, for each $y \in J$, where $\mu \in I$. If $\lambda \in I, \mathscr{F} \in \mathbf{F}(J)$, and $\psi \mathscr{F} \stackrel{q_{\lambda}}{\longrightarrow} x$, then $\kappa \sigma \mathscr{F} \stackrel{q_{r(\lambda, \mu)}}{\longrightarrow} x$. 
If $(X, \mathbf{q})$ is a p.c.s. which satisfies $K_{T}$ (respectively, $\left.F_{T}\right)$ relative to a given $t$-norm $T$, then the triple $(X, \mathbf{q}, T)$ is called a Kowalsky (respectively, Fischer) probabilistic convergence space. For brevity, we abbreviate these terms k.p.c.s. and f.p.c.s., respectively. The first proposition summarizes some elementary facts about these notions.

PROPOSITION 3.2. (a) Everyf.p.c.s. is a k.p.c.s.

(b) If $\mathbf{q}_{p}$ is a constant p.c.s., then for any $t$-norm $T,(X, \mathbf{q}, T)$ is a k.p.c.s. (respectively, f.p.c.s.) if and only if the convergence structure $p$ satisfies $K$ (respectively, $F$ ).

(c) If $(X, \mathbf{q}, T)$ is a k.p.c.s. (respectively, f.p.c.s.) and $T^{\prime}$ is a $t$-norm such that $T^{\prime} \leq T$, then $\left(X, \mathbf{q}, T^{\prime}\right)$ is k.p.c.s. (respectively, f.p.c.s.).

(d) If $(X, \mathbf{q})$ is a topological p.c.s., then $(X, \mathbf{q}, T)$ is a f.p.c.s. for any $t$-norm $T$.

(e) If $(X, \mathbf{q})$ is a p.c.s. such that $q_{\lambda}=\left(q_{\lambda}\right)_{K}$ holds for all $\lambda \in I$, where $\left(q_{\lambda}\right)_{K}$ is the finest convergence structure on $X$ coarser than $q_{\lambda}$ which satisfies $K$ and $q_{\lambda}=(\mathbf{q})_{\lambda}$, then $(X, \mathbf{q}, T)$ is a k.p.c.s. for any $t$-norm $T$.

An example of a k.p.c.s. which is not a f.p.c.s. is the constant k.p.c.s. $\left(Y, \mathbf{q}_{p}, T\right)$, where $(Y, p)$ is the convergence space of Example 1.9 and $T$ is any $t$-norm.

If a p.c.s. $(X, \mathbf{q})$ has a p.c.s. property (for example, regular, left-continuous), any k.p.c.s. or f.p.c.s. of the form $(X, \mathbf{q}, T)$ is said to have the same property.

In Section 1, we observed that property $F$ (being equivalent to 'topological') is an initial property, whereas property $K$ is not. In the setting of p.c.s.'s, property $F_{T}$ is not equivalent to being topological (as we shall see latter), but it is an initial property if the $t$-norm $T$ remains fixed.

PROPOSITION 3.3. For a given $t$-norm $T$, let $\left\{\left(Y_{\alpha}, \mathbf{p}^{\alpha}, T\right): \alpha \in A\right\}$ be a set of f.p.c.s.'s. Let $X$ be a set and let $f_{\alpha}: X \rightarrow Y_{\alpha}$ be a function, for each $\alpha \in A$. If $\mathbf{q}$ is the initial structure on $X$ relative to the families $\left\{\left(Y_{\alpha}, \mathbf{p}^{\alpha}\right): \alpha \in A\right\}$ and $\left\{f_{\alpha}: \alpha \in A\right\}$, then $(X, \mathbf{q}, T)$ is a f.p.c.s.

PROOF. By our previous characterization of initial structures for convergence spaces in Section $1,(\mathbf{q})_{\lambda}=q_{\lambda}$ where, for each $\lambda \in I, \mathscr{F} \stackrel{q_{\lambda}}{\longrightarrow} x$ if and only if $f_{\alpha}(\mathscr{F}) \stackrel{p_{\lambda}}{\longrightarrow}$ $f_{\alpha}(x)$, for all $\alpha \in A$. It remains to show that $(X, \mathbf{q}, T)$ satisfies $F_{T}$. Assume $\psi: J \rightarrow X, \sigma: J \rightarrow \mathbf{F}(X)$ such that $\sigma(z) \stackrel{q_{\mu}}{\longrightarrow} \psi(z)$, for each $z \in J$, and $\psi \mathscr{F} \stackrel{q_{\lambda}}{\longrightarrow} x$, where $\lambda \in I$. Let $\alpha \in A$, and let $\psi^{*}=f_{\alpha} \circ \psi, \sigma^{*}(z)=f_{\alpha}(\sigma(z))$, for all $z \in J$, and $\psi^{*}(\mathscr{F})=f_{\alpha}(\psi \mathscr{F})$. Since $p^{\alpha}$ satisfies $F_{T}, \kappa \sigma^{*} \mathscr{F} \rightarrow f_{\alpha}(x)$ in $\left(Y_{\alpha}, p_{T(\alpha, \mu)}^{\alpha}\right)$. Since this holds for all $\alpha \in A$ and $f_{\alpha}(\kappa \sigma \mathscr{F})=\kappa \sigma^{*} \mathscr{F}, \kappa \sigma \mathscr{F} \rightarrow x$ in $\left(X, q_{T(\alpha, \mu)}\right)$, and it follows that $(X, \mathbf{q}, T)$ is a f.p.c.s.

The next proposition and its proof are analogous to Proposition 1.8, so the proof is omitted. 
PROPOSITION 3.4. For a given t-norm $T$, let $\left\{\left(Y_{\alpha}, \mathbf{p}^{\alpha}, T\right): \alpha \in A\right\}$ be a set of k.p.c.s.'s. Let $X$ be a set, and let $f_{\alpha}: X \rightarrow Y_{\alpha}$ be an injective function, for each $\alpha \in A$. If $\mathbf{q}$ is the initial structure on $X$ relative to the families $\left\{\left(Y_{\alpha}, \mathbf{p}^{\alpha}\right): \alpha \in A\right\}$ and $\left\{f_{\alpha}: \alpha \in A\right\}$, then $(X, \mathbf{q}, T)$ is a k.p.c.s.

By making some obvious alterations, one can use Example 1.9 to show that $K_{T}$ is not a p.c.s. initial property.

From the two preceding propositions, it follows that for any p.c.s. $(X, \mathbf{q})$ and any $t$-norm $T$, there is a finest p.c.s. $\mathbf{q}_{K_{T}}$ coarser than $\mathbf{q}$ such that $\left(X, \mathbf{q}_{K_{T}}, T\right)$ is a k.p.c.s., and a finest p.c.s. $\mathbf{q}_{F_{T}}$ coarser than $\mathbf{q}$ such that $\left(X, \mathbf{q}_{F_{T}}, T\right)$ is a f.p.c.s.; these are called the $K_{T}$ and $F_{T}$ modifications of $(X, \mathbf{q}, T)$, respectively. For any $t$-norm $T$, it is clear that $\mathbf{q}_{F_{T}} \leq \mathbf{q}_{K_{T}} \leq \mathbf{q}$.

Proposition 3.5. If $(X, \mathbf{q}, T)$ is a f.p.c.s., then $(X, \mathbf{q})$ is pretopological.

Proof. For fixed $\lambda \in I$, let $\left\{\mathscr{H}_{\alpha}: \alpha \in J\right\}$ be the set of all ultrafilters on $X$ which $q_{\lambda}$-converge to $x$. Define $\psi: J \rightarrow X$ by $\psi(\alpha)=x$, and let $\sigma(\alpha)=\mathscr{H}_{\alpha}$, for each $\alpha \in J$. If $\mathscr{F}=\{J\}$, then $\psi \mathscr{F}=\dot{x} \stackrel{q_{1}}{\longrightarrow} x$, and hence $\kappa \sigma \mathscr{F} \rightarrow x$ in $\left(X, q_{T_{(1 . .)}}\right)$. But $T(1, \lambda)=\lambda$ by Definition 3.1, so $\kappa \sigma \mathscr{F} \stackrel{q_{\lambda}}{\longrightarrow} x$. But $\bigcap_{z \in J} \sigma(z)=\bigcap_{\alpha \in J} \mathscr{H}_{\alpha}=\mathscr{V}_{q_{\lambda}}(x)$, so $\mathscr{V}_{q_{\lambda}}(x) \stackrel{q_{\lambda}}{\longrightarrow} x$. Since this holds for all $x \in X$, each $q_{\lambda}$ is a pretopology.

PROPOSITION 3.6. If $(X, \mathbf{q}, T)$ is a pretopological k.p.c.s., and $T(\lambda, \lambda)=\lambda$, for all $\lambda \in I$, then $(X, \mathbf{q})$ is topological.

PROOF. It suffices to show, for arbitrary $\lambda \in I$ and $A \subseteq X$, that $c l_{q_{\lambda}}^{2} A=c l_{q_{\lambda}} A$. Assume $x \in c l_{q_{\lambda}}^{2} A$. Let $\mathscr{F}$ be an ultrafilter on $X$ such that $\mathscr{F} \stackrel{q_{\lambda}}{\longrightarrow} x$ and $c l_{q_{\lambda}} A \in \mathscr{F}$. For each $y \in c l_{q_{\lambda}} A$, let $\mathscr{H}_{y}$ be an ultrafilter which contains $A$ and $q_{\lambda}$-converges to $y$. Define

$$
\sigma(y)= \begin{cases}\dot{y}, & y \notin c l_{q_{\lambda}} A \\ \mathscr{H}_{y}, & y \in c l_{q_{\lambda}} A\end{cases}
$$

then $A \in \bigcap\left\{\sigma(y): y \in c l_{q_{\lambda}} A\right\}$ and hence $A \in \kappa \sigma \mathscr{F}$, which $T_{(\lambda, \lambda)}$-converges to $x$ by $K_{T}$. By the assumption $T(\lambda, \lambda)=\lambda, x \in c l_{q_{\lambda}} A$, and hence $c l_{q_{\lambda}}^{2} A=c l_{q_{\lambda}} A$, which completes the proof.

COROLLARY 3.7. If $(X, \mathbf{q}, T)$ is a f.p.c.s. and $T(\lambda, \lambda)=\lambda$, for all $\lambda \in I$, then $(X, \mathbf{q}, T)$ is topological.

The next three propositions give necessary and sufficient conditions for a pretopological p.c.s. equipped with a $t$-norm to be a f.p.c.s. 
PROPOSITION 3.8. Let $(X, \mathbf{q})$ be a pretopological p.c.s. and let $T$ be a $t$-norm. Then $(X, \mathbf{q}, T)$ is a f.p.c.s. if and only if the following condition $(*)$ is satisfied:

(*) For arbitrary $\lambda, \mu \in T$ and for each $V \in \mathscr{V}_{q_{T(\lambda, \mu)}}(x)$, there exists $W \in \mathscr{V}_{q_{\lambda}}(x)$ such that, for each $y \in W, V \in \mathscr{V}_{q_{\mu}}(y)$.

ProOF. Assume that $(X, \mathbf{q}, T)$ satisfies $F_{T}$ and let $V \in \mathscr{V}_{p}(x)$, where $p=q_{T(\hat{i}, \mu)}$. Suppose that for each $W \in \mathscr{V}_{q_{\lambda}}(x)$, there exists $y_{w} \in W$ such that $V \notin \mathscr{V}_{q_{\mu}}\left(y_{w}\right)$. Let $J=\left\{y_{w}: W \in \mathscr{V}_{q_{\lambda}}(x)\right\}$, and let $\mathscr{F}$ be the filter of sections of the net $\left(y_{w}\right)_{W \in \mathscr{V}_{q_{\lambda}}(x)}$. Define $\psi(z)=z$ and $\sigma(z)=\mathscr{V}_{q_{\mu}}(z)$, for all $z \in J$. Thus $\sigma(z) \stackrel{q_{\mu}}{\longrightarrow} \psi(z)=z$. It follows by $F_{T}$ that $\kappa \sigma \mathscr{F} \stackrel{p}{\longrightarrow} x$, which implies $\kappa \sigma \mathscr{F} \geq \mathscr{V}_{p}(x)$. Thus $V \in \kappa \sigma \mathscr{F}$, and so there is $F \in \mathscr{F}$ such that $V \in \bigcap\{\sigma(y): y \in F\}$. Let $y_{w} \in F$, then $V \in \sigma\left(y_{w}\right)$. However, $\sigma\left(y_{w}\right)=\mathscr{V}_{q_{\mu}}\left(y_{w}\right)$, and by assumption $V \notin \mathscr{V}_{q_{\mu}}\left(y_{w}\right)$, a contradiction. Thus there exists $W \in \mathscr{V}_{q_{\mathrm{\lambda}}}(x)$ such that, for each $y \in W, V \in \mathscr{V}_{q_{u}}(y)$.

Conversely, assume the given condition. To verify $F_{T}$, assume that: $J$ is a set, $\psi: J \rightarrow X, \sigma: J \rightarrow \mathbf{F}(X)$ is such that $\sigma(y) \stackrel{q_{\mu}}{\longrightarrow} \psi(y)$ for each $y \in J$, and $\mathscr{F} \in$ $\mathbf{F}(J)$ is such that $\psi \mathscr{F} \stackrel{q_{\lambda}}{\longrightarrow} x$. It remains to show that $\kappa \sigma \mathscr{F} \stackrel{p}{\longrightarrow} x$ (or equivalently, $\left.\kappa \sigma \mathscr{F} \geq \mathscr{V}_{p}(x)\right)$, where $p=q_{T(\lambda, \mu)}$. If $V \in \mathscr{V}_{p}(x)$, there exists (by Condition (*)) $W \in \mathscr{V}_{q_{\lambda}}(x)$ such that, for each $y \in W, V \in \mathscr{V}_{q_{\mu}}(y)$. Since $\psi \mathscr{F} \geq \mathscr{V}_{q_{\lambda}}(x), W \in \psi \mathscr{F}$, and so there exists $F \in \mathscr{F}$ such that $\psi(F) \subseteq W$. Let $y \in F$; then $\psi(y) \in W$, and therefore $V \in \mathscr{V}_{q_{\mu}}(\psi(y))$. Since $\sigma(y) \geq \mathscr{V}_{q_{\mu}}(\psi(y)), V \in \sigma(y)$ and it follows that $V \in \bigcap\{\sigma(y): y \in F\}$. Thus $V \in \kappa \sigma \mathscr{F}$, which establishes that $\kappa \sigma \mathscr{F} \geq \mathscr{V}_{p}(x)$.

In convergence space theory, it is well known that for a convergence space $(X, q)$, $V \in \mathscr{V}_{q}(x)$ if and only if $x \notin c l_{q}(X-V)$. Under the assumption of Proposition 3.8, one can use this fact to reformulate Condition $(*)$ in the following equivalent form:

(**) For every $\mu, \lambda \in I$ and $A \in X, c l_{q_{\lambda}}\left(c l_{q_{\mu}}(A)\right) \subseteq c l_{p}(A)$, where $p=q_{T(\lambda, \mu)}$.

COROllary 3.9. Let $(X, \mathbf{q})$ be a pretopological p.c.s. and let $T$ be a $t$-norm. Then $(X, \mathbf{q}, T)$ is a f.p.c.s. if and only if Condition (**) is satisfied.

PROPOSITION 3.10. Let $(X, \boldsymbol{q})$ be a pretopological p.c.s. and let $T$ be a $t$-norm. Then $(X, \mathbf{q}, T)$ is a f.p.c.s. if and only if $(X, \mathbf{q}, T)$ is a k.p.c.s.

PROOF. It remains only to show that every pretopological k.p.c.s. $(X, \mathbf{q}, T)$ is a f.p.c.s., and this can be achieved by verifying Condition (**). If $x \in c l_{q_{\lambda}}\left(c l_{q_{\mu}}(A)\right)$, then there exists $\mathscr{G} \stackrel{q_{\lambda}}{\longrightarrow} x$ for which $c l_{q_{\mu}} A \in \mathscr{G}$. Choose, for each $G \in \mathscr{G}, x_{G} \in G \cap c l_{q_{\mu}} A$, and let $\mathscr{F}$ denote the filter of sections of the net $\left(x_{G}\right)_{G \in \mathscr{G}}$. Since $\mathscr{F} \geq \mathscr{G}, \mathscr{F} \stackrel{q_{\lambda}}{\longrightarrow} x$. Moreover, $x_{G} \in c l_{q_{\mu}} A$ implies the existence of $\mathscr{H}_{G} \stackrel{q_{\mu}}{\longrightarrow} x_{G}$ such that $A \in \mathscr{H}_{G}$, for 
all $G \in \mathscr{G}$. We define $\sigma: X \rightarrow \mathbf{F}(X)$ by:

$$
\sigma(z)= \begin{cases}\mathscr{H}_{G}, & \text { if } z=x_{G} \text { for } G \in \mathscr{G} \\ \dot{z}, & \text { otherwise }\end{cases}
$$

Then $\kappa \sigma \mathscr{F} \stackrel{p}{\longrightarrow} x$ (where $p=q_{T(\lambda, \mu)}$ ), since $K_{T}$ is satisfied. Fix a base element $F_{o}=\left\{x_{G}: G \subseteq G_{o}, G \in \mathscr{G}\right\}$ in $\mathscr{F}$. Then $\bigcap_{z \in F_{o}} \sigma(z)=\bigcap\left\{\mathscr{H}_{G}: G \subseteq G_{o}, G \in \mathscr{G}\right\}$, and therefore $A \in \bigcap_{z \in F_{o}} \sigma(z)$. It follows that $A \in \kappa \sigma \mathscr{F}$, and this implies $x \in c l_{p} A$, which establishes Condition (**).

Combining Corollary 3.7 and Proposition 3.10, we obtain the following analog to Corollary 1.6 .

COROLLARY 3.11. Let $(X, \mathbf{q})$ be a pretopological p.c.s. and let $T$ be a $t$-norm such that $T(\lambda, \lambda)=\lambda$, for all $\lambda \in I$. The following statements are equivalent:

(a) $(X, \mathbf{q})$ is topological; $(b)(X, \mathbf{q})$ satisfies $K_{T} ;(c)(X, \mathbf{q})$ satisfies $F_{T}$.

We now return to the three examples of Section 2. In the next example, we show that a f.p.c.s. can fail to be topological if the $t$-norm fails to satisfy the condition of Corollary 3.11.

EXAMPLE 3.12. Let $(X, q)$ be the p.c.s. defined in Example 2.3. Recall that for arbitrary $r \in \mathbf{C}(X)$,

$$
q_{\lambda}= \begin{cases}\iota, & \lambda=0 \\ r, & 0<\lambda \leq 1 / 2 \\ \delta, & 1 / 2<\lambda \leq 1 .\end{cases}
$$

We examine the diagonal properties of this p.c.s. relative to two different $t$-norms.

CASE 1. $T(\lambda, \mu)=\max \{\lambda+\mu-1,0\}$. By considering four cases, one can show that $(X, \mathbf{q}, T)$ is a k.p.c.s. for any convergence structure $r \in \mathbf{C}(X)$. Thus, by Proposition 3.10, $(X, \mathbf{q}, T)$ is a f.p.c.s. if and only if $r$ is any pretopology. In particular, we see that a k.p.c.s. need not have the property that every $(q)_{\lambda}$ is a limit structure (compare with Proposition 1.7), and a f.p.c.s. need not be topological.

CASE 2. $T_{1}(\lambda, \mu)=\min \{\lambda, \mu\}$. One can show that $\left(X, \mathbf{q}, T_{1}\right)$ is a k.p.c.s. if and only if $(X, r)$ satisfies Condition $K$ of Section 1. In particular, $r$ must be a limit structure. It follows from Proposition 3.11 that $\left(X, q, T_{1}\right)$ is a f.p.c.s. if and only if $r$ is a topology.

The next example shows that the p.c.s. of Example 2.4 (based on convergence almost everywhere) fails to satisfy $K_{T}$ for any $t$-norm $T$. This example suggests that requiring all p.c.s.'s to satisfy a diagonal condition (as in [3]) would be undesirable. 
EXAMPLE 3.13. Let $(X, \mathbf{q})$ be the p.c.s. of Example 2.4. We shall show that $(X, \mathbf{q})$ fails to satisfy $K_{T}$ for any $t$-norm $T$.

Choose $\lambda=\mu=1$; by Definition $3.1, T(1,1)=1$, for any $t$-norm $T$. Let $\mathscr{F}$ be any filter on $X q_{1}$-converging to 0 (the zero function) such that $\mathscr{F}$ has a nested base $\left\{F_{n}: n \in N\right\}$ such that the cardinality of $F_{n}-F_{n+1}$ is $c$, the cardinality of $I$. Then, for each $n \in N$, there is a bijection $g_{n}: F_{n}-F_{n+1}$ onto [0,1]. Define $\sigma: X \rightarrow \mathbf{F}(X)$ such that $\sigma(y) \stackrel{q_{1}}{\longrightarrow} y$ for each $y \in X$, and $\sigma(y)\left(g_{n}(y)\right) \stackrel{\tau}{\longrightarrow} 1$, when $y \in F_{n}-F_{n+1}$, for each $n \in N$.

Assume that $\kappa \sigma \mathscr{F} \stackrel{q_{1}}{\longrightarrow} 0$. Then there exists $A \subseteq I$ of Lebesque measure 0 and $\kappa \sigma \mathscr{F}(\alpha) \stackrel{\tau}{\longrightarrow} 0$, for each $\alpha \in I-A$. Let $\alpha \in I-A$ be fixed; then there is some $m \in N$ and $B \in \bigcap\left\{\sigma(y): y \in F_{m}\right\}$ such that $B(\alpha) \subseteq[0,1 / 2]$. However for $y=g_{m}^{-1}(\alpha) \in F_{m}-F_{m+1}, \sigma(y)\left(g_{m}(y)\right) \stackrel{t}{\longrightarrow} 1$, which is a contradiction since $B(\alpha) \in \sigma(y)\left(g_{m}(y)\right)$ implies that $[0,1 / 2] \in \sigma(y)\left(g_{m}(y)\right)$. Thus $\kappa \sigma \mathscr{F}$ fails to $q_{1^{-}}$ converge to 0 , and $K_{T}$ fails for any $t$-norm $T$.

EXAMPLE 3.14. Let $(Y, \mathbf{p})$ be the p.c.s. discussed in Example 2.5. Recall that $Y$ is the set of all real-valued, Lebesgue measurable functions on $R$, with functions identified which are equal almost everywhere, and $p_{\lambda}$ is defined on $Y$ for $\lambda \in(0,1]$ as follows: $\mathscr{F} \stackrel{p_{\lambda}}{\longrightarrow} f$ if and only if, for each $g \in F, l\{\mu \in I:|g(\mu)-f(\mu)|<a\} \geq \epsilon$. We showed in Example 2.5 that $\mathbf{p}$ is pretopological and first countable, and that $p_{1}$ is convergence in probability. We shall now show that $(Y, \mathbf{p})$ satisfies $F_{T}$ relative to the $t$-norm $T(\lambda, \mu)=\max \{\lambda+\mu-1,0\}$.

Assume $\psi: J \rightarrow Y, \sigma: J \rightarrow \mathbf{F}(Y)$ with $\sigma(y) \stackrel{p_{\mu}}{\longrightarrow} \psi(y)$ for all $y \in J$, and $\psi \mathscr{F} \stackrel{p_{\lambda}}{\longrightarrow} f$. Given $a>0$ and $0<\epsilon<T(\mu, \lambda)=\lambda+\mu-1$, choose $\alpha$ and $\beta$ such that $0<\alpha<\mu, 0<\beta<\lambda$, and $\alpha+\beta-1 \geq \epsilon$. Since $\psi \mathscr{F} \stackrel{p_{\mu}}{\longrightarrow} f$, there is $F \in \mathscr{F}$ such that, for each $g \in F, l\{\eta \in I:|\psi(g)(\eta)-f(\eta)|<a / 2\} \geq \alpha$. Similarly, $\sigma(g) \stackrel{p_{\lambda}}{\longrightarrow} \psi(g)$ implies there is $A_{g} \in \sigma(g)$ such that, for each $h \in A_{g}$, $l\{\eta \in I:|h(\eta)-\psi(g)(\eta)|<a / 2\} \geq \beta$. Define $A=\bigcup\left\{A_{g}: g \in F\right\}$; then $A \in \kappa \sigma \mathscr{F}$. Recall that $l(C \cup D)=l(C)+l(D)-l(C \cap D) \geq l(C)+l(D)-1$ for any subsets $C, D$ of $I$. Since $\{\eta \in I:|h(\eta)-\psi(g)(\eta)|<a / 2\} \cap\{\eta \in I:|\psi(g)(\eta)-f(\eta)|<a / 2\} \subseteq$ $\{\eta \in I:|h(\eta)-f(\eta)|<a\}$, we see that $l\{\eta \in I:|h(\eta)-f(\eta)|<a\} \geq \beta+\alpha-1 \geq \epsilon$, for each $h \in A$. It follows that $\kappa \sigma \mathscr{F} \rightarrow f$ in $\left(Y, p_{T(\hat{\lambda . \mu})}\right)$, and therefore $(Y, \mathbf{p}, T)$ is a f.p.c.s.

A probabilistic closure operator cl (see Definition 2.15) will be called an $F_{T^{-}}$probabilistic closure operator if it satisfies the following condition $(* *)$ relative to a $t$-norm $T$ :

(**) For each $\lambda, \mu \in I$ and $A \subseteq X, \operatorname{cl}(\operatorname{cl}(A, \mu), \lambda) \subseteq \operatorname{cl}(A, T(\lambda, \mu))$.

If $\mathbf{c l}=\mathbf{c l}_{\mathbf{q}}$ for some p.c.s. $\mathbf{q}$ on $X$ with $t$-norm $T$, the Condition (**) as just defined coincides with Condition $(* *)$ as defined prior to Corollary 3.9 . 
Our final results establish a bijection between the p.c.s.'s on $X$ satisfying $F_{T}$ and the $F_{T}$-probabilistic closure operators on $X$.

PROPOSITION 3.15. For any f.p.c.s. $(X, \mathbf{q}, T)$, the associated probabilistic closure operator $\mathbf{c l}_{\mathbf{q}}$ satisfies Condition (**). Furthermore, assume $\mathbf{c l}$ is any probabilistic closure operator satisfying (**) for some $t$-norm $T$, and let $\mathbf{p}$ be the pretopological p.c.s. such that $(\mathbf{p})_{\lambda}$ is the pretopology determined by the closure operator $c_{\lambda}$, where $c l_{\lambda}(A)=\operatorname{cl}(A, \lambda)$, for all $A \subseteq X$ and $\lambda \in I$. Then $(X, \mathbf{p}, T)$ is a f.p.c.s.

PROOF. If $(X, \mathbf{q}, T)$ is a f.p.c.s., then $\mathbf{c l}_{\mathbf{q}}$ satisfies $(* *)$ by Corollary 3.9. Conversely, let $\mathbf{c l}$ be any probabilistic closure operator satisfying (**) for some $t$-norm $T$, and let $\mathbf{p}$ be the pretopological p.c.s. determined by $\mathbf{c l}$ as indicated. Then it follows immediately from Proposition 2.16 that $\mathbf{c l}=\mathbf{c l}_{\mathbf{p}}$, and the conclusion that $(X, \mathbf{q}, T)$ is an f.p.c.s. follows by Corollary 3.9.

COROLlary 3.16. If $(X, \mathbf{q}, T)$ is an f.p.c.s., then the correspondence $\mathbf{q} \leftrightarrow \mathbf{c l}_{\mathbf{q}}$ is a bijection between the set of all p.c.s.'s on $X$ satisfying $F_{T}$ and the set of all $F_{T}$-probabilistic closure operators on $X$.

Consider a triple $(X, \mathrm{cl}, T)$, where $X$ is a set, $T$ a $t$-norm on $X$, and cl a $F_{T}$-closure operator. Frank [4] and Florescu [3] both identify such a triple as a 'probabilistic topological space' (although Frank's definition of the closure operator appears to be more general than ours). We wish to point out, in view of Case 1 of Example 3.12, that the f.p.c.s. corresponding to such a space need not be topological.

\section{Acknowledgement}

The authors are grateful to Professors B. Schweizer and H. Sherwood for bringing to our attention the article by L. C. Florescu. Subsequently, Professor Florescu provided us with further relevant material.

\section{References}

[1] K. L. Chung, A course in probability theory (Academic Press, New York, 1974).

[2] H. R. Fischer, 'Limesräume', Math. Ann. 137 (1959), 269-303.

[3] L. C. Florescu, 'Probabilistic convergence structures', Aequationes Math. 38 (1989), 123-145.

[4] M. J. Frank, 'Probabilistic topological spaces', J. Math. Anal. and Appl. 34 (1971), 67-81.

[5] D. C. Kent, 'Convergence groups and convergence uniformities', Fund. Math. 60 (1967), 213-222.

[6] — 'Decisive convergence spaces, Fréchet spaces, and sequential spaces', Rocky Mountain J. Math. 1 (1971), 367-374. 
[7] H. J. Kowalsky, 'Limesräume und Komplettierung', Math. Nachr. 12 (1954), 301-340.

[8] B. Schweizer and A. Sklar, Probabilistic metric spaces (North-Holland, New York, 1983).

[9] H. Sherwood, 'On E-spaces and their relation to other classes of probabilistic spaces', J. London Math. Soc. 44 (1969), 441-448.

Department of Mathematics

University of Central Florida

Orlando, FL 32816

USA
Department of Pure and Applied Mathematics

Washington State University

Pullman, WA 99164-3113

USA 